This item was submitted to Loughborough's Research Repository by the author.

Items in Figshare are protected by copyright, with all rights reserved, unless otherwise indicated.

\title{
Spatio-temporal attention deep recurrent Q-network for POMDPs
}

PLEASE CITE THE PUBLISHED VERSION

https://doi.org/10.1007/978-3-030-30241-2_9

PUBLISHER

Springer

VERSION

AM (Accepted Manuscript)

PUBLISHER STATEMENT

This is a pre-copyedited version of a contribution published in Progress in Artificial Intelligence: 19th EPIA Conference on Artificial Intelligence, EPIA 2019. edited Paulo Moura Oliveira • Paulo Novais • Luís Paulo Reis (Eds.) published by Springer. The definitive authenticated version is available online via https://doi.org/10.1007/978-3-030-30241-2_9

\section{LICENCE}

CC BY-NC-ND 4.0

\section{REPOSITORY RECORD}

Etchart, Mariano, Pawel Ladosz, and David Mulvaney. 2019. "Spatio-temporal Attention Deep Recurrent Qnetwork for Pomdps". figshare. https://hdl.handle.net/2134/11197976.v1. 


\title{
Work in Progress: Spatio-Temporal Attention Deep Recurrent Q-Network for POMDPs
}

\author{
Authors removed for blind review \\ Institute removed for blind review
}

\begin{abstract}
One of the long-standing challenges for reinforcement learning agents is to deal with noisy environments. Although progress has been made in producing an agent capable of optimizing its environment in fully observable conditions, partial observability still remains a difficult task. In this paper, a background review of the sub-field of partial observability is made and a novel model is proposed which inspired by human perception, utilizes two fundamental machine learning concepts, attention and memory, to better confront a noisy environment.
\end{abstract}

\section{Introduction}

Successful control policies may be obtained through reinforcement learning (RL), an agent's framework to learning by interacting in an environment. Traditionally, RL is difficult to train and recent progress - made possible by a few tricks and the combination of deep learning [8] - has funnelled a large amount of attention to the field because of the ability to provide human-like performance [13]. Yet within the field, there exists several challenges that plague the RL communities. One of the prominent challenges is extending these control policies from relatively simple environments to more dynamic and noisy environments, owning to the nature of the real-world.

There is far less research within RL of partial observability, one of the stepping stones which would allow an agent to extend beyond these simple environments and begin to deal with the noisy states and systems of the world. A large portion of research papers assume the RL problem to be fully observable, formalized as a Markov Decision Process [2]. Although Markov decision processes provide an adequate formalization of the reinforcement learning problem, when approaching real-world complexity, MDPs lack representing some of the features of how an agent sees an environment including partially observable states and redundant information. Having noted that performance drops and human-level performance has not yet been achieved in the games domain for partially observable environments, this paper proposes a model to operate under partially obersvable MDPs (POMDPs) by taking inspiration from human perception and proposes a combinational attention model (spatial and temporal) with memory to move towards a more robust agent who is better suited to handle nosier situations.

Previous approaches to POMDPs with deep reinforcement learning have managed to improve previous benchmarks in the Atari domain, although few have been specifically inspired by human cognition. To the knowledge of the authors, there have not been work on deep RL for POMDP with spatio-temporal attentional mechanisms. Variants of spatio-temporal attention have been used before for several applications including action recognition [19,23,18], caption generation and image classification [1], video recognition [4] and object tracking [25] and many more although have never been used for solving POMDPs before.

Given the vast amount of research on the additive nature and synergic importance of spatial with temporal attention for human perception [3] in the cognitive neuroscience domain, one naturally wonders how this could be applied to an agent in Atari games in a noisy environment. This study hypothesizes that formulating an agent with a spatio-temporal attention mechanism for partially observable environments will perform more robustly and additively benefit from both mechanisms.

\subsection{Partially Observable Markov Decision Processes}

As an MDP represents the RL problem, Partially Observable Markov Decision Processes (POMDP) represent a partially observable environment. Two variables are introduced to the MDP tuple, a set of 
observations $\Omega$ and the observation function $\mathrm{O}$ which connects the observations with the states. The probability of observing an observation $o \in \Omega$ in state $\mathrm{s}$ is $\mathrm{O}(\mathrm{s}, \mathrm{o})$, and the POMDP can be summarized by the tuple $\{\mathrm{S}, \mathrm{A}, \mathrm{R}, \mathrm{P}, \Omega, \mathrm{O}\}$. In an POMDP, the environment only exhibits observations $\Omega$ and the states $\mathrm{S}$ are unknown. To create an POMDP for Atari games, one may simulate a POMDP by occulting a game screen with some probability. This technique was used in a study by Hausknecht et al. where a POMDP was created in the game of Pong with every game screen having 0.5 probability of being fully observable or completely occulted.

\subsection{Related Works in POMDPs}

POMDPs are known to be difficult problems to solve since an observation is not enough information to select an action and multiple observations are required to capture elements of the underlying state. In Hausknecht's study [5], a Deep Recurrent Q-Network (DRQN) is proposed and demonstrated to outperform DQN in partially observable MDPs. This is due to the correlations captured in the DRQN's recurrent neural network with long-short term memory cells and gives the agent an ability to integrate information through time to more robustly handle partial observability. Similarly the action-specific deep recurrent Q-network [24] uses an RNN with an additional network, to keep track of the influence of certain observations over others. Both these recent models form part of approaches which utilize RNNs to encode the past in order to deal with noise and occulted observations.

On the other hand, Igl et. al. argue that learning a policy in a partially observable environment with a Blackbox approach, such as in an RNN, can lead to a high reliance on these encodings of the past and that this implicit inference can lead to a less optimal solution for a complex environment [6]. Thus, it introduces a generative model of the environment and uses this to provide inference in the missing information. The approach uses the combination of encoding history in RNN like in DRQN or ADRQN, but also using belief inference with a variational autoencoder with the argument that model-based and model free learning must be utilized together.

Other research into POMDPs assume transition probabilities, reward models and observation functions are known which is not the case when working towards real-world environments [16, 15].

\subsection{Deep Recurrent Q-Network}

DQN's inability to deal with POMDPs was improved by the Deep Recurrent Q-Network from Hausknecht et al. [5] by adding an LSTM due to its ability to encode the history of past states and maintain longer term decencies between states. It implicitly maintains a history of previous state through its 3 gate structure (input, output and forget gate) determining which input features are more important than others. These additions to the network allow it to outperform DQN in partially observable environments, in the case of flickering Atari game screens. For this reason, the authors chose this as a starting point to deal with the problem of partial observability.

Model Architecture and Implementation The DRQN architecture replaces the second-last fully connected layer in the DQN with a long-short term memory (LSTM) layer instead. This leads to an aspect of 'unrolling' the timesteps of the LSTM and backpropagation through time [21] must be used instead. Fundamentally, DQN and DRQN receive the input differently whereby DQN takes 4 frames at once and DRQN only a single frame. Although because of the aforementioned unrolling the DRQN maintains a history of the past 4 states (or for however long it is chosen to unroll).

The DRQN and DQN trains by sampling experience tuples from a replay buffer [13] consisting of state, reward, action, and next state from one interaction with the environment.

The entire DRQN is trained end-to-end including the LSTM layer, the loss function is the same as DQNs except for the added parameters of the LSTM layer:

$$
L_{i}\left(\theta_{i}\right)=E_{\left(s_{t}, r_{t}, a_{t}, s_{t+1}\right) \sim D}\left[\left(\left(r_{t}+\gamma \max _{a^{\prime}} \hat{Q}\left(s_{t+1}, a_{t}^{\prime} \mid \theta_{i}\right)-Q\left(s_{t}, a_{t} \mid \theta_{i}\right)\right)\right]\right.
$$


Where $\theta$ is the weights and biases of the network, $\mathrm{E}$ is the set of experiences whereby $\mathrm{D}$ indicates the replay memory, $\hat{Q}$ is the $\mathrm{Q}$-function of the target network and $\left(r_{t}+\gamma \max _{a^{\prime}} \hat{Q}\left(s_{t+1}, a_{t}^{\prime} \mid \theta_{i}\right)\right.$ represents the update target and $Q\left(s_{t}, a_{t} \mid \theta_{i}\right)$ the current Q-value estimate. The optimizer tries to minimize this function in each iteration of training.

The deep recurrent Q-network (DRQN) was implemented by extending an already existing implementation of DQN. The RNN was unrolled for 4 timesteps. Refer to appendix for the entire network body.

\subsection{Spatial Attention Model for POMDP}

Visual attention [22] is a technique that allows an agent to focus on more relevant aspects of an input image and can therefore learn a policy associated with it more quickly because learning updates are more information efficient (outlined in Background section). This paper therefore introduces visual attention to the DRQN in order to exploit the fact that the entire spatial input is rarely needed to successfully learn policies, it is usually a very important subsection of the image that provides the information needed for learning. This is particularly true in some Atari games where the majority of the game screen is irrelevant or for asthetics and only a small portion of the screen becomes essential to the decision making. Not only does this enable the agent to converge faster but increases the chances of learning correct policies.

Model Architecture and Implementation The DRQN is augmented following an attention mechanism inspired by the Deep Attention Q-Network [20]. Soft attention uses the weighted sum of an attention matrix and the current input matrix in order to exemplify certain sections of the image more than others.

The attention network $\mathrm{g}$ is placed after the convolutional layers and before the LSTM. The input image is transformed into a set of vectors and then fed into the $G$ network which consists of two linear layers and a SoftMax activation function. The output of the network may be summarized as such:

$$
g\left(v_{t}^{i}, h_{t-1}\right)=\operatorname{softmax}\left(\operatorname{linear}\left(\operatorname{Tanh}\left(\operatorname{Linear}\left(v_{t}^{i}\right)+W h_{t-1}\right)\right)\right)
$$

Where Linear layers are affine transformations, $v_{t}^{i}$ represents the input vectors of the image, $h_{t-1}$ is the previous hidden state of the LSTM and W is a weight matrix with no bias. The output is then weighted and summed in order to find the context vector:

$$
z_{t}=\sum_{i=1}^{L} g\left(v_{t}^{i}, h_{t-1}\right) * v_{t}^{i}
$$

This simple addition allows the model to retain its differentiability and may still be trained end-toend, therefore the loss function is the same as the one in DRQN with a larger $\theta$ to include additional parameters in the attention network.

\subsection{Spatio-Temporal Model for POMDPs}

Temporal attention performs the same selective approach as seen in spatial attention but in the time domain, choosing certain timesteps over others. Although there is a smaller body of research focusing on temporal attention in the machine learning community $[25,17,11]$, it is well-established in the neuroscientific community that the combination of spatial and temporal attention in human perception is a critical aspect of perceiving occulted and moving objects in time [3] [10], two for the most prominent features of a flickering Atari game screen. The authors combine spatial and temporal attention in an attempt to recreate the additive effects [10] seen in human perception, in a deep RL agent. 


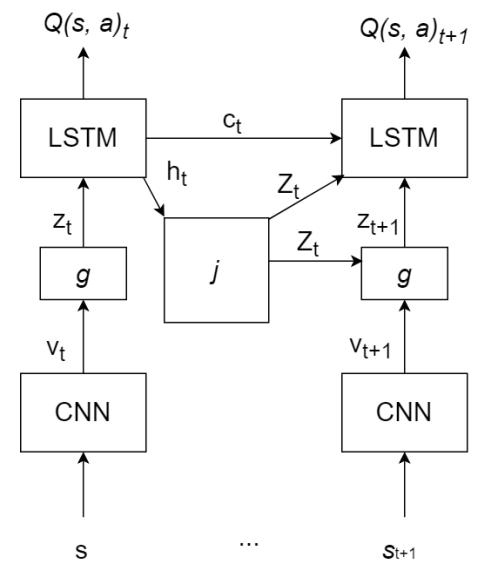

Fig. 1. Spatio-Temporal Attention DRQN Netowrk

Model Architecture and Implementation An attention network $J$ is added in between the LSTM's timesteps to optimize for the most important states of the past history. In DRQN state are implicitly discriminated by the LSTM through its 3 gates, with the addition of the $\mathrm{J}$ network, states are being explicitly discriminated through the weights matrix of the attention network.

The output of a LSTM is a hidden state which logically feeds into itself when unrolling the timesteps. During the unrolling, each hidden state is given an equal weight in a normal LSTM or in the DRQN, however in this case the hidden state is fed into the attention network before taking the weighted sum to find the context vector and then outputted to the LSTM for the next timestep as shown schematically in Figure 6 . The output of the $\mathrm{J}$ network is as follows:

$$
j\left(h_{t-1}\right)=\operatorname{softmax}\left(\operatorname{Tanh}\left(\text { Linear }\left(h_{t-1}\right)+W h_{t-1}\right)\right.
$$

Where linear is an affine transformation with no bias, $h_{t-1}$ represents the previous hidden state, and $\mathrm{W}$ is a weight matrix. The context vector is then thus calculated as the weighted sum:

$$
z_{t}=\sum_{i=1}^{L} j\left(h_{t-1}\right) * h_{t-1}
$$

The resulting model has spatial attention network $\mathrm{G}$ and temporal attention network $\mathrm{J}$ as shown in Figure 1

\subsection{Training, Evaluation and Hyperparameters}

On the ALE, policies were evaluated after 50,000 steps of training for 25,000 steps with an epsilon-greedy policy with epsilon equal to 0.05 and the average reward per episode was calculated for this evaluation period. During training time polices took 50,000 exploration steps before beginning to train with an e-greedy policy of epsilon value of 1 annealed to 0.2 over 1 million steps. A discount factor of 0.99 was used and an asynchronous replay buffer size of 100k with batch size of 32 . The optimizer used was Adam [7] with learning rate 0.0001. Target networks were updated every 1000 steps, and 32 steps were sampled from the replay buffer to train the agent roughly every 4 steps taken in the environment. Networks were frozen during evaluation periods.

In all models 'smooth L1' loss was used which acts as a way to clip the error to improve stability in training as mentioned in the DQN paper.

Flickering Pong In the flickering domain, almost all agents performed poorly as seen in previous studies. The spatial attention model and spatio-temporal attention model however performed marginally better than the DRQN, with the DRQN being unable to reach past a score of - 15 ever and maintaining 
under -19 for the majority of epochs as seen in Figure 2. Spatial attention showed more instability in the training than the other model with large oscillations. The spatio-temporal model managed to achieve the highest reward of the three in the same number of epochs.

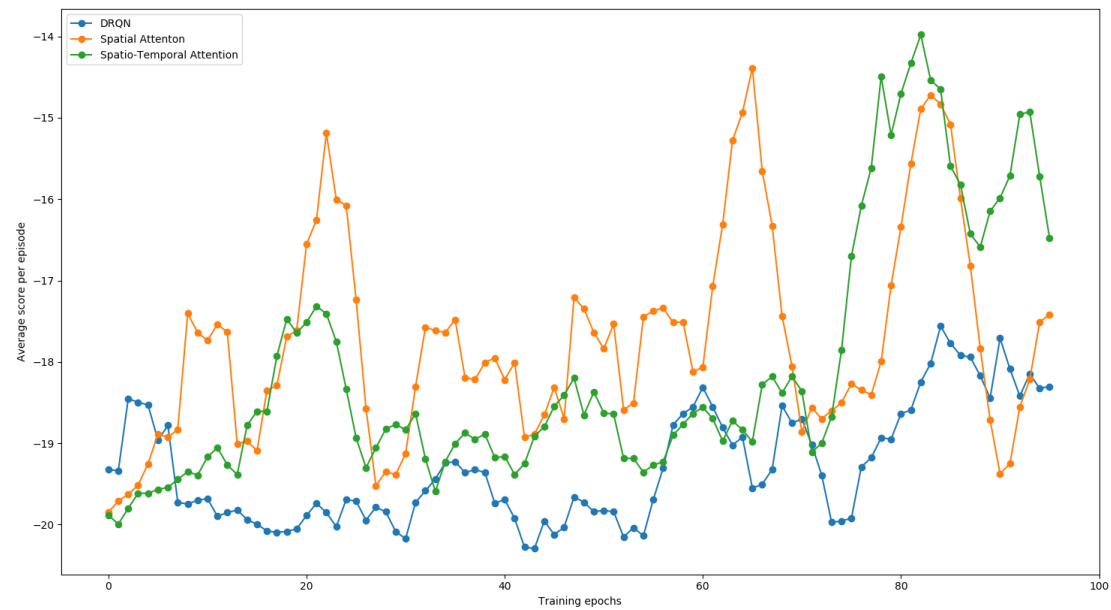

Fig 2. Comparitive moving average of all models on Flickering Pong

\section{Fully Observable Pong}

In fully observable, the agent seemed to perform worse than the DRQN. The improvement in performance of spatial attention does not match the normal rate of convergence of an algorithm such as DRQN.
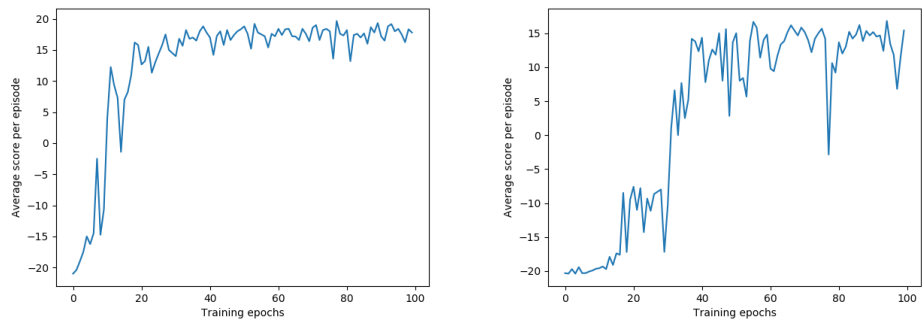

Fig 3. From left to right, DRQN and DRQN with Spatial Temporal Attention in MDPs

\section{Conclusions}

In this paper, a review of the RL literature for POMDPs was done, as well as a proposal for a novel model with attention and memory mechanisms. It was showed that the performance of the model was marginally better than the other model in a partially observable version of Pong, although did not show any improvements in the fully observable case. Since none of the algorithms manage to converge in the flickering domain for less than 5 million steps, more trials would have to be carried in order to ascertain that attention is key for partial observability. The authors are currently working towards the following directions 1) experimenting with extended tests covering 100 million steps or more, so that to ensure agents converge and the varying complexity of the environments is not a factor, 2) employing different environments such as those which are inherently partially observable since flickering a screen may not in fact adequately represent a noisy state since they are either fully observable or fully occulted, 3) Adopting other techniques such as neural episodic learning [14] where very valuable experience tuples are replayed multiple times. Other approaches with hard attention [12] or global approaches [9] may be taken. 


\section{References}

1. Ba, J., Grosse, R.B., Salakhutdinov, R.R., Frey, B.J.: Learning wake-sleep recurrent attention models. In: NIPS (2015)

2. Bellman, R.E.: A markovian decision process. In: MDP (1957)

3. Doherty, J.R., Rao, A., Mesulam, M.M., Nobre, A.C.: Synergistic effect of combined temporal and spatial expectations on visual attention. The Journal of neuroscience : the official journal of the Society for Neuroscience $25(2005)$

4. Fu, Y., Wang, X., Wei, Y., Huang, T.S.: Sta: Spatial-temporal attention for large-scale video-based person re-identification. CoRR abs/1811.04129 (2019)

5. Hausknecht, M.J., Stone, P.: Deep recurrent q-learning for partially observable mdps. In: AAAI Fall Symposia (2015)

6. Igl, M., Zintgraf, L.M., Le, T.A., Wood, F., Whiteson, S.: Deep variational reinforcement learning for pomdps. In: ICML (2018)

7. Kingma, D.P., Ba, J.: Adam: A method for stochastic optimization. CoRR abs/1412.6980 (2015)

8. LeCun, Y., Bengio, Y., Hinton, G.E.: Deep learning. Nature 521, 436-444 (2015)

9. Luong, T., Pham, H.Q., Manning, C.D.: Effective approaches to attention-based neural machine translation. In: EMNLP (2015)

10. MacKay, A., Juola, J.F.: Are spatial and temporal attention independent? Perception and psychophysics $\mathbf{6 9}$ 6, 972-9 (2007)

11. Meng, L., Zhao, B., Chang, B., Huang, G., Tung, F., Sigal, L.: Where and when to look? spatio-temporal attention for action recognition in videos. CoRR abs/1810.04511 (2018)

12. Mnih, V., Heess, N., Graves, A., Kavukcuoglu, K.: Recurrent models of visual attention. In: NIPS (2014)

13. Mnih, V., Kavukcuoglu, K., Silver, D., Rusu, A.A., Veness, J., Bellemare, M.G., Graves, A., Riedmiller, M.A., Fidjeland, A., Ostrovski, G., Petersen, S., Beattie, C., Sadik, A., Antonoglou, I., King, H., Kumaran, D., Wierstra, D., Legg, S., Hassabis, D.: Human-level control through deep reinforcement learning. Nature 518, 529-533 (2015)

14. Pritzel, A., Uria, B., Srinivasan, S., Badia, A.P., Vinyals, O., Hassabis, D., Wierstra, D., Blundell, C.: Neural episodic control. In: ICML (2017)

15. Roijers, D.M., Whiteson, S., Oliehoek, F.A.: Point-based planning for multi-objective pomdps. In: IJCAI (2015)

16. Ross, S., Pineau, J., Paquet, S., Chaib-draa, B.: Online planning algorithms for pomdps. The journal of artificial intelligence research 32 2, 663-704 (2008)

17. Song, J., Guo, Z., Gao, L., Liu, W., Zhang, D., Shen, H.T.: Hierarchical lstm with adjusted temporal attention for video captioning. In: IJCAI (2017)

18. Song, S., Lan, C., Xing, J., Zeng, W., Liu, J.: Spatio-temporal attention-based lstm networks for 3d action recognition and detection. IEEE Transactions on Image Processing 27(7), 3459-3471 (July 2018). https://doi.org/10.1109/TIP.2018.2818328

19. Song, S., Lan, C., Xing, J., Zeng, W., Liu, J.: An end-to-end spatio-temporal attention model for human action recognition from skeleton data (2017)

20. Sorokin, I., Seleznev, A., Pavlov, M., Fedorov, A., Ignateva, A.: Deep attention recurrent q-network. CoRR abs/1512.01693 (2015)

21. Werbos, P.J.: Backpropagation through time: What it does and how to do it (1990)

22. Xu, K., Ba, J., Kiros, R., Cho, K., Courville, A.C., Salakhutdinov, R.R., Zemel, R.S., Bengio, Y.: Show, attend and tell: Neural image caption generation with visual attention. In: ICML (2015)

23. Yan, S., Smith, J., Lu, W., Zhang, B.: Hierarchical multi-scale attention networks for action recognition. Signal Processing Image Communication 61 (08 2017). https://doi.org/10.1016/j.image.2017.11.005

24. Zhu, P., Li, X., Poupart, P., Miao, G.: On improving deep reinforcement learning for pomdps. CoRR abs/1704.07978 (2017)

25. Zhu, Z., Wu, W., Zou, W., Yan, J.: End-to-end flow correlation tracking with spatial-temporal attention. 2018 IEEE/CVF Conference on Computer Vision and Pattern Recognition pp. 548-557 (2018) 